need to automate EWSS before implementing it at a large scale to decrease workload, record duplication, and score calculation errors. More efforts need to be carried out in staff training, motivation, and support as they are key aspects towards success.

\section{REDUCING IV INFILTRATION AND POTENTIAL HARM IN THE PEDIATRIC EMERGENCY DEPARTMENT - KASCH}

Milfi Al-Onazi, Omar Burhish, Rahayu Abd Rashid, Faith Vabaza, Sherfa Sakiral, Mari Leizl Villanueva. Emergency Care Center, King Abdullah Specialized Children's Hospital, Riyadh

\subsection{6/bmjoq-2019-PSF.59}

Background There was a significant increase in intravenous (IV) infiltration in the month of March 2016 in the Pediatric Emergency Department - KASCH. The Acute Care team noted an increase in IV-related incidence and there were 30 generated safety reporting system (SRS) reports from January to March 2016. This prompted the unit leaders to create a taskforce in order to review the causes and the factors leading to incidence and causing patient harm.

Methods Using the PDSA (plan-do-study-act) quality model, the quality improvement team collected data generated through the SRS on IV infiltration/extravasation. The reports were analyzed as to the causes and harm of the incident. Using a cause and effect diagram, the team identified the root causes as non-adherence to the insertion and sterility technique, staff competency on IV insertion and monitoring, type of dressing used, lighting, and lack of guidelines to support the practice. In order to draw a reliable conclusion, several PDSA cycles were tested and implemented: (1) data collection and audit tool design; (2) staff education and standardized documentation; (3) formulation of the escalation process and guidelines; and (4) continuous monitoring and auditing of IV infiltration/extravasation and regular reporting to the daily key performance indicator (KPI).

Results For the initial throughput of the project, the IV Watchers on the Move Team noted a marked decrease in infiltration in August 2018 as evidenced by the nurses' compliance in IV infiltration prevention strategies - i.e., (1) hourly IV site checks, (2) mandatory use of smart pumps, and (3) timely and accurate documentation of IV assessment.

Conclusion The IV Watchers on the Move QI Project was able to design a mechanism to reduce the potential harm caused by infiltration. Part of the intervention was the hourly assessment and early recognition of impending infiltration. Nurses were instructed to be cautious when administering highly concentrated medications. With all of the efforts and initiative shown throughout, the pediatric patients benefited from the successful interventions that finally led to the safe delivery of nursing care.

\section{EFFECTIVENESS OF PROCEDURE EXPLANATION IN REDUCING ANXIETY FOR PATIENTS UNDERGOING MAGNETIC RESONANCE IMAGING}

Ali Aldhebaib, Oinam Gokulchandra Singh, Jayachandran Vetrayan, Meshal Al-Aqeel, Abdulmajeed Modhi Alotaibi, Fahad Namshan Aldosari, Abdulhadi Alqhutani, Homed Jaber M Alghamde. Radiology/College of Applied Medical Sciences, King Saud Bin Abdulaziz University for Health Sciences

10.1136/bmjoq-2019-PSF.60
Background Magnetic resonance imaging (MRI) is potentially considered as the greatest invention in the world. In addition, all the people in the medical field have been surprised by the capability of MRI in the past 25 years. There has been a massive increase in the use of MRI in the clinical field. More than 80 million MRI procedures occur every year around the globe. Patients who are undergoing MRI examinations infrequently experience anxiety as a consequence of the procedure or the environment. Reducing patient anxiety is considered as one of the most common concerns affecting the outcome of the MRI scan. The purpose of this study was to investigate the effectiveness of procedure explanation on controlling anxiety levels in adolescent patients undergoing MRI.

Methods The Institutional Review Board (IRB) ethics committee approved this cross-sectional study. Convenient sampling techniques were used to select 37 adolescent patients (19 females and 18 male) who attended the MRI appointment unit at the Medical Imaging Department of King Abdulaziz Medical City, Riyadh, Saudi Arabia. Three tools were used to collect data: the sociodemographic data sheet, patient assessment knowledge sheet, and the StateTrait Anxiety Inventory (STAI) questionnaire. STAI is a self-report test designed to measure patient anxiety level; 40 questions are divided into two subcategories; 20 state (defined as fear, nervousness, discomfort, etc, and how the person feels right now), and 20 traits (defined as stress, worry, discomfort, etc, that the person experiences on a daily basis).

Results The state anxiety level showed a statistically significant difference in patients' knowledge between the pre-test and post-test with or without instruction. For the group with instruction, their anxiety level significantly reduced. The trait anxiety level showed no significant difference between pre-test and post-test with or without instruction.

Conclusion Because of lack of awareness about the procedural instructions for the patient before undergoing MRI, it may affect the procedure prognosis and outcome. Our suggestion for the future is to increase awareness about MRI and to improve communication skills of MRI staff to educate the patient in a good way to reduce patient anxiety.

\section{SUCCESSFUL IMPROVEMENT IN POST-EXPOSURE OUTCOMES AMONG HEALTHCARE WORKERS EXPOSED TO VACCINE-PREVENTABLE DISEASES IN A HOSPITAL SETTING}

Abubaker Omer Fadlilmula, Dina Afurong, Diana Alagano, Aiman El-Saed Majid Alshamrani, Hanan Balkhy. Infection Prevention and Control, National Guard Health Affairs - Riyadh

\subsection{6/bmjoq-2019-PSF.61}

Background Healthcare workers (HCWs) working in hospitals are at higher risk of exposure to patients with different infectious agents, particularly measles, mumps, rubella, and varicella. Identifying the HCWs who are at risk and initiating post-exposure management is critical to reduce the risk of further spread to other patients and HCWs. This is especially important in high- risk hospital areas such as emergency departments. The objective of the current study was to assess the impact of a multi- partner intervention on the outcomes of post-exposure management. 